\title{
Worse inflammatory profile in omnivores than in vegetarians associates with the gut microbiota composition
}

\author{
Ana Carolina Franco-de-Moraes ${ }^{1}$, Bianca de Almeida-Pititto ${ }^{2}$, Gabriel da Rocha Fernandes ${ }^{3}$, \\ Everton Padilha Gomes ${ }^{4}$, Alexandre da Costa Pereira ${ }^{4}$ and Sandra Roberta G. Ferreira ${ }^{1 *}$
}

\begin{abstract}
Aims: To describe the abundance of major phyla and some genera in the gut microbiota of individuals according to dietary habits and examine their associations with inflammatory markers, insulin resistance, and cardiovascular risk profile.

Methods: A total of 268 non-diabetic individuals were stratified into groups of dietary types (strict vegetarians, lactoovo-vegetarians, and omnivores). The taxonomic composition and phylogenetic structure of the microbiota were obtained through the analysis of the 16S rRNA gene. Samples were clustered into operational taxonomic units at 97\% similarity using GreenGenes 13.5 database. Clinical, biochemical, and circulating inflammatory markers were compared by ANOVA or Kruskal-Wallis test.

Results: The sample (54.2\% women, mean age 49.5 years) was composed of 66 strict vegetarians, 102 lactoovo-vegetarians and 100 omnivores. Considering the entire sample, the greatest abundant phyla were Firmicutes $(40.7 \pm 15.9 \%)$ and Bacteroidetes (39.5 $\pm 19.9 \%)$, and no difference in abundances was found between individuals with normal and excess weight. Stratifying by dietary types, the proportion of Firmicutes was lower and of Bacteroidetes was higher in strict vegetarians when compared to lacto-ovo-vegetarians and omnivores. At the genus level, strict vegetarians had a higher Prevotella abundance and Prevotella/Bacteroides ratio than the other groups. They also had a lower proportion of Faecalibacterium than lacto-ovo-vegetarians, and both vegetarian groups had higher proportions than did omnivores. Succinivibrio and Halomonas from the Proteobacteria phylum were overrepresented in omnivores. The omnivorous group showed higher values of anthropometric data, insulin, HOMA-IR, and a worse lipid profile. Inflammatory markers exhibited a gradual and significant increase from the vegetarians and lacto-ovovegetarians to the omnivorous group.

Conclusions: There are differences in gut microbiota composition of individuals with distinct dietary habits, who differ according to their inflammatory and metabolic profiles. Based on the findings relative to bacteria abundances and on their recognized actions in the metabolism, we suggest that exposure to animal foods may favor an intestinal environment which could trigger systemic inflammation and insulin resistance-dependent metabolic disorders.
\end{abstract}

Keywords: Gut microbiota, Diet, Biomarkers, Inflammation, Insulin resistance

\footnotetext{
*Correspondence: sandrafv@usp.br

1 Department of Epidemiology, School of Public Health, University of Sao

Paulo, Av. Dr. Arnaldo, 715, Sao Paulo, SP Zip code 01246-904, Brazil

Full list of author information is available at the end of the article
} 


\section{Background}

The role of dietary habits for risk and for protection against cardiometabolic diseases is largely recognized [1]. While a high-fat low-fiber western diet has been associated with type 2 diabetes mellitus, dyslipidemia and cardiovascular disease, a vegetarian diet with a beneficial cardiometabolic profile and lower rates of cardiovascular events [2, 3]. Moreover, the ability of diet to decrease all-cause and cardiovascular mortality was demonstrated prospectively in vegetarians from the adventist health study-2 [4]. It is known that the body fat accumulation is an underlying mechanism of cardiometabolic disease by triggering a low-grade inflammation and insulin resistance $[5,6]$. More recently, there is a growing interest in the participation of gut bacteria mediating the dietinduced cardiometabolic risk [7-9]. Animal studies have contributed to understanding how bacterial communities influence energy extraction, fat deposition, inflammatory status and insulin sensitivity [10-13].

In humans, numerous factors like delivery type, breastfeeding, antibiotic use and dietary habits have been implicated in the interindividual gut microbiota variability and diseases risk [14-18]. In contrast to the initial studies in lean and obese animals [11], metagenomic analyses have indicated that the identification of relative abundance of the two major phyla, Firmicutes and Bacteroidetes, may not be sufficient to predict body adiposity and obesity-related diseases [19].

Inconsistent results regarding the initially described increased Firmicutes/Bacteroidetes ratio (F/B ratio) in human obesity have motivated deepening the knowledge of the phylogenetic structure of microbial communities in population exposed to different environments [20-22]. Studies in some populations have detected associations of dietary patterns with the microbiota composition and certain chronic diseases [21, 23-25]. Underlying pathways of these associations are not completely clarified but it is recognized the ability of a high-fat diet to increase intestinal permeability allowing lipopolysaccharides access in bloodstream [26-28]. A gut microbiomeinduced endotoxemia has been associated with increased risk of type 2 diabetes mellitus [7, 13, 29].

Scarce data are available in South America countries characterized by a large variety of foods and dietary habits. The study ADVENTO-Analysis of diet and lifestyle for cardiovascular prevention in 7th-day Adventists (http://www.estudoadvento.org), conducted in a sample of the Brazilian population, has represented a unique opportunity to investigate how vegetarianism and exposure to animal food are associated with the gut microbiota and cardiometabolic risk profile. We hypothesized that dietary-dependent microbial composition influences inflammatory status, insulin resistance, and cardiovascular risk in individuals undergoing distinct dietary habits.

The aims of this study were: to describe the abundance of major phyla and genera in the gut microbiota of nondiabetic Brazilians classified according to dietary types (strict vegetarian, lacto-ovo-vegetarian, and omnivore); and to compare their inflammatory status, insulin resistance index, and cardiovascular risk profile.

\section{Methods \\ Subjects}

A convenience sample of first 300 participants from the ADVENTO (http://www.estudoadvento.org), aged 35-65 years old, was invited to join in this cross-sectional analysis. Exclusion criteria were body mass index (BMI) $\geq 40 \mathrm{~kg} / \mathrm{m}^{2}$, diabetes mellitus, history of inflammatory bowel diseases or persistent diarrhea, and use of antibiotics or probiotic or prebiotic supplements within the 2 months prior to data collection. A total of 268 subjects satisfied those criteria and were stratified according to dietary type: strict vegetarian, lacto-ovo-vegetarian, and omnivore. Participants were examined at the Investigation Center of the University of Sao Paulo Hospital from March 2013 to October 2014. After overnight fasting, they visited the Center for clinical examination and biological samples collection.

A trained staff collected dietary and clinical data. Dietary types were defined based on the referred food consumption for the last year. Participants were considered strict vegetarians when consuming no animal product (red meat, poultry, fish, eggs, milk, and dairy products $<1$ time/month); lacto-ovo-vegetarians when consuming dairy products and/or eggs $\geq 1$ time/month, but no fish or meat (red meat, poultry, and fish $<1$ time/month); and omnivores if they eat animal products (red meat, poultry, fish, eggs, milk, and dairy products) more than once a month [30, 31].

\section{Clinical data}

Height was obtained using a fixed stadiometer and weight with subjects wearing light clothing and no footwear, placed on a digital scale with $200 \mathrm{~kg}$ capacity, accurate to the nearest $100 \mathrm{~g}$. BMI was calculated as weight in kilograms divided by height in meters squared; values $\geq 25.0 \mathrm{~kg} / \mathrm{m}^{2}$ was considered weight excess. Waist circumference was measured at the midpoint between the bottom of the rib cage and the top of the iliac crest during minimal respiration. The body composition was measured using a bioelectrical impedance analysis (BIA-InBody230; BioSpace, Seoul, Korea). Blood pressure was taken using a validated oscillometric device (Omron HEM 705CPINT, Omron Health Care, Lake Forest, IL, USA) after a 5-min rest in a sitting position. Three measurements were taken at 1 -min intervals. The 
mean of the blood pressure measurements was used in analysis. Hypertension was defined as blood pressure $\geq 140 / 90 \mathrm{mmHg}$ (either systolic or diastolic) or use of antihypertensive medication.

\section{Analytical methods}

Blood samples were collected while fasting and during a 2-h standard 75-g oral glucose tolerance test, and were immediately centrifuged for plasma glucose and lipid determinations. Categories of glucose tolerance were defined according to the American Diabetes Association criteria [32]. Aliquots were frozen at $-80{ }^{\circ} \mathrm{C}$ for further determinations of insulin and inflammatory markers (C-reactive protein-CRP, lipopolysaccharides-LPS, interleukin-10-IL-10, tumor necrosis factor-alphaTNF- $\alpha$ and E-selectin). Insulin resistance was estimated using homeostasis model assessment (HOMA-IR) [33]. The HOMA-IR has been largely used as an insulin resistance surrogatein clinical and epidemiological studies, based on its strong correlation with the estimates obtained by the euglycaemic clamp [33-35].

The ratio TNF- $\alpha / \mathrm{IL}-10$ was calculated as index of inflammatory response [36].

Plasma glucose was measured by the hexokinase method (ADVIA Chemistry; Siemens, Deerfield, IL, USA). Plasma insulin was determined by enzyme-linked immunosorbent assay (ELISA) (Siemens, Deerfield, IL, USA). Total and high-density lipoprotein cholesterol (HDL-c) and triglycerides were measured by enzymatic colorimetric assay (ADVIA Chemistry; Siemens, Deerfield, IL, USA), while low-density lipoprotein cholesterol (LDL-c) was calculated by the Friedewald equation. High-sensitivity CRP was determined by immunochemistry (Dade Behring; Siemens, IL, USA), and LPS using an ELISA kit (My Bio Source, San Diego, CA, USA). Concentrations IL-10, TNF- $\alpha$ and E-selectin were simultaneously determined by the Multiplex ${ }^{\circledR}$ (R\&D Systems, Minnesota, MN, USA).

\section{Gut microbiota}

Fecal samples were maintained under refrigeration $\left(6^{\circ} \mathrm{C}\right)$ within a maximum of $24 \mathrm{~h}$ after collection, and then the aliquots were stored at $-80^{\circ} \mathrm{C}$ until analysis. DNA was extracted using the Maxwell ${ }^{\circledR} 16$ DNA purification kit and the protocol carried out in the Maxwell ${ }^{\circledR} 16$ Instrument according to the manufacturer's instructions (Promega, Madison, WI, USA). Taxonomic composition and phylogenetic structure of a microbial community were obtained through the analysis of the 16S rRNA gene using the Illumina ${ }^{\circledR}$ MiSeq platform and the V4 region. DNA library construction and sequencing were performed following the manufacturer's instruction (Illumina, San Diego, CA, USA), and the workflow described by Caporaso et al. [37]. Samples were clustered into operational taxonomic units (OTUs) at 97\% similarity with Qiime v1.8 using the GreenGenes 13.5 database.

\section{Statistical analysis}

Descriptive data were expressed as means and standard deviations or medians and interquartile range. Since distributions of some variables were skewed, they were logtransformed before analysis to achieve normality; some values in tables were back-transformed to return to the natural scale. Groups of participants classified according to the three dietary types were compared by ANOVA and Bonferroni post hoc test, or by the Kruskal-Wallis test when indicated. Statistical analyses were carried out with IBM SPSS Statistics, version 22.

Participants were also stratified into two groups, omnivores and non-omnivores (strict plus lacto-ovo vegetarians), and the DESeq2 package was used for comparisons [38]. The package provides $\log 2$ fold changes attributable to a given variable over the mean of normalized counts of OTUs. The log2 fold changes represent the comparison against the reference level, which is $\log 2$ (Omnivores/ Non-omnivores). If differences between omnivores and non-omnivores are equal to zero, it indicates that there is no difference between the means of the groups.

\section{Results}

Among 268 participants, 54.2\% were women and 41.4\% had weight excess. Stratifying according to dietary type, 66 were strict vegetarians, 102 lacto-ovo-vegetarians, and 100 omnivores, and groups did not differ according to male-to-female ratios and age $(49.6 \pm 8.5,49.6 \pm 8.6$, $49.1 \pm 8.2$ years, $\mathrm{p}=0.878$, respectively). The frequencies of weight excess [26\% (95\% CI 15-37) versus 38\% (95\% CI $29-47$ ) versus $55 \%$ (95\% CI $45-65)$, pre-diabetes [ $21 \%$ (95\% CI 11-31) versus 29\% (95\% CI 20-38) and 36\% (95\% CI 27-45)], and hypertension [18\% (95\% CI 9-27) versus $26 \%$ (95\% CI 17-34) and 33\% (95\% CI 24-43)] were higher in the omnivores than in lacto-ovo-vegetarians and strict vegetarians, respectively.

Thirteen phyla were identified and five were present in all the fecal samples analyzed. Large relative abundances of Firmicutes $(40.7 \pm 15.9 \%)$ and Bacteroidetes $(39.5 \pm 19.9 \%)$ were found (Fig. 1), followed by Proteobacteria. Stratifying by dietary type, the proportion of Firmicutes (Fig. 1, panel a) was lower and of Bacteroidetes was higher in the strict vegetarian group (Fig. 1, panel b) when compared to lacto-ovo-vegetarians and omnivores. No differences were found in the comparisons of the $\mathrm{F} / \mathrm{B}$ ratio between subsets of participants with normal and weight excess, considering the whole sample as well as within each dietary type.

The abundance of OTUs according to dietary types was also compared at genus level. Strict vegetarians had 

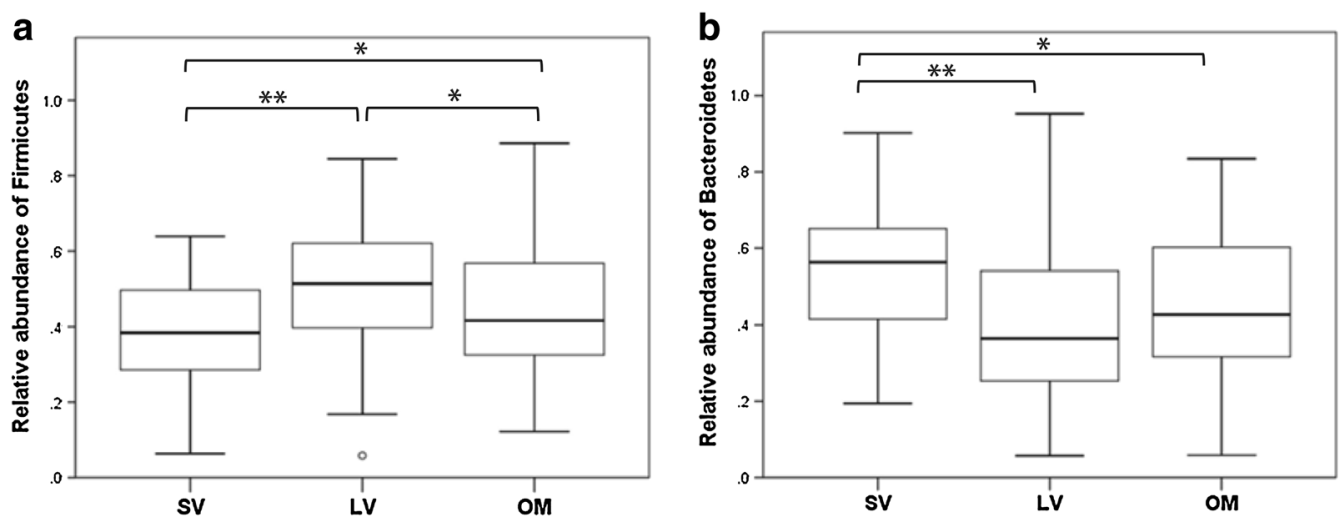

Fig. 1 Relative abundance of Firmicutes (a) and Bacteroidetes (b) according to dietary types. Central lines of the box plots are the means; box outline equals 1 SD; the bar denotes 2 SD. SV strict vegetarian, LV lacto-ovo-vegetarian, OM omnivore. ANOVA followed by Bonferroni post hoc test. ${ }^{*} p<0.05,{ }^{* *} p<0.005$

higher rates of Prevotella genus that belongs to the Bacteroidetes phylum (Fig. 2, panel a), and a higher Prevotella/Bacteroides ratio $(8.4 \pm 0.3)$ than the other groups.
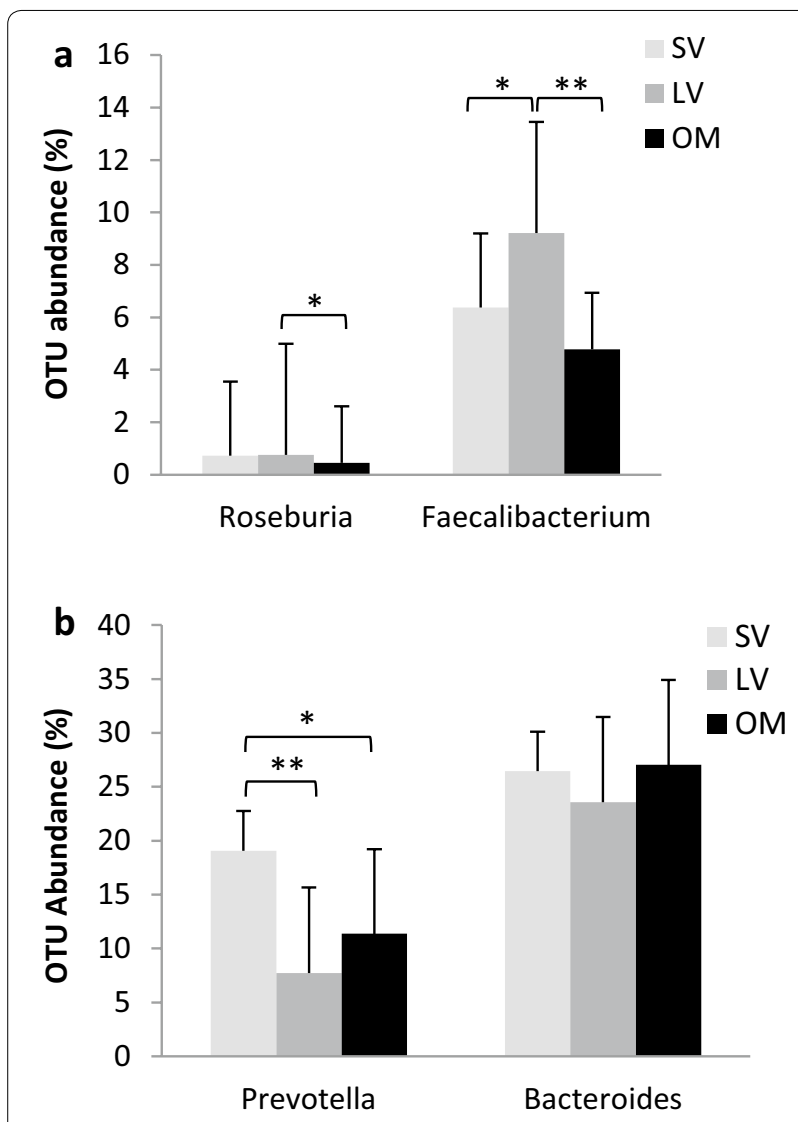

Fig. 2 Mean values of abundance (\%) of genera belonging to the major phyla. a Firmicutes phylum: Roseburia and Faecalibacterium genera. b Bacteroidetes phylum: Prevotella and Bacteroides genera. ANOVA followed by Bonferroni post hoc test. ${ }^{*} p<0.05$, ${ }^{* *} p<0.005$
On the other hand, strict vegetarians had a lower proportion of Faecalibacterium genus from the Firmicutes phylum than lacto-ovo-vegetarians, and both vegetarian groups had higher proportions than in omnivores (Fig. 2, panel b).

Differences of selected OTUs between omnivores and non-omnivores (strict plus lacto-ovo-vegetarians), members of phyla Firmicutes, Proteobacteria, and Actinobacteria, assessed by the DESeq2, were shown in Fig. 3. The omnivore group showed much higher abundances of genera Succinivibrio and Halomonas, which belong to Proteobacteria, while non-omnivores had more OTUs from Actinobacteria and Roseburia genus from Firmicutes, compared to the counterparts.

The omnivorous group showed higher mean values of anthropometric data and worse lipid profile than the others (Table 1). Non-significant increases in fasting plasma glucose was verified across the groups, but omnivores exhibited higher insulin and HOMA-IR values $(\mathrm{p}<0.001)$. Medians of CRP, LPS, and TNF- $\alpha / \mathrm{IL}-10$ ratio increased gradually from the vegetarian toward the omnivorous group.

\section{Discussion}

The hypothesis that diet should influence systemic inflammatory status, insulin resistance and cardiovascular risk profile, via gut microbiota composition, is supported in the present study. In a developing country population of South America, the association between dietary habits and abundance of certain bacterial genera was demonstrated. Non-diabetic Brazilians undergoing distinct dietary types showed that vegetarians had a more favorable gut microbiota composition, characterized by less Firmicutes and more Bacteroidetes than omnivores. Furthermore, among the Firmicutes there was a predominance of genera associated with beneficial phenotypes, 


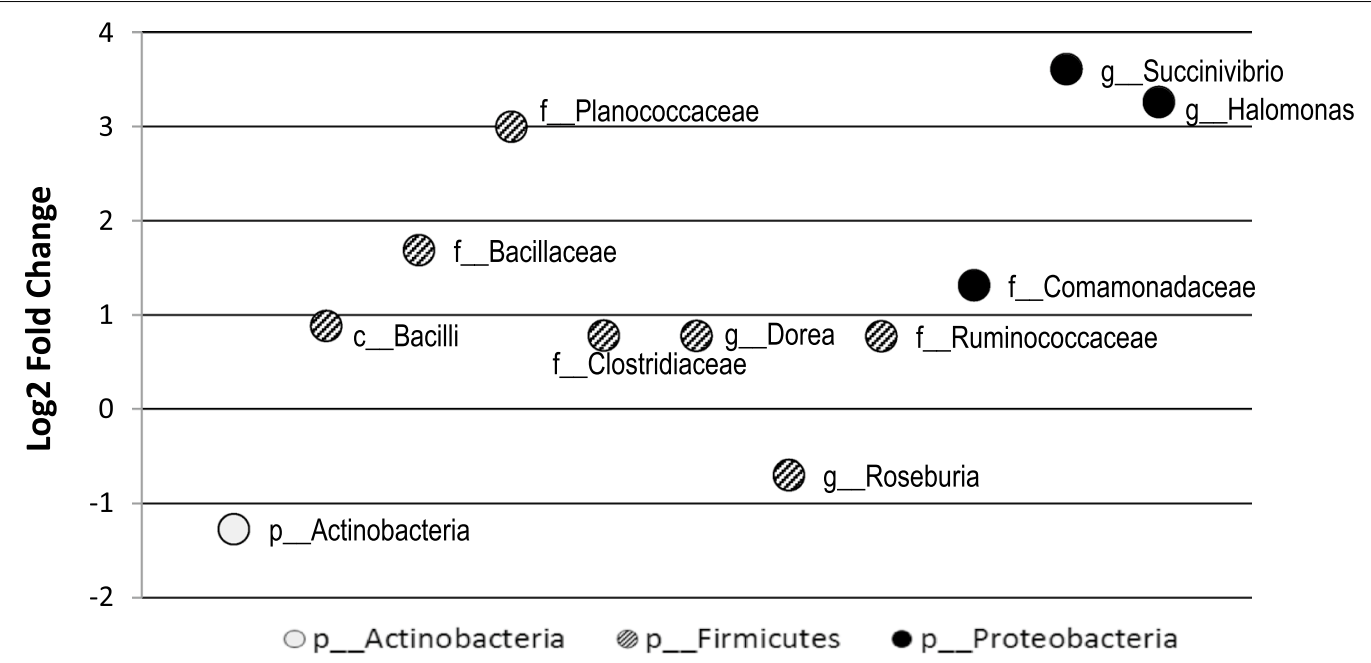

Fig. 3 Log2 fold changes in mean reads of selected OTUs in omnivore and non-omnivorous group (strict plus lacto-ovo-vegetarians) at the deepest level identified. DESeq2 detected significant differences between dietary types at $p<0.005$. Bacteria represented in circles at different colors belong to different phyla

Table 1 Mean (standard deviation) or medians (interquartile range) of clinical data of participants according to dietary type

\begin{tabular}{|c|c|c|c|c|}
\hline & $\begin{array}{l}\text { Strict vegetarian } \\
\mathrm{N}=66\end{array}$ & $\begin{array}{l}\text { Lacto-ovo-vegetarian } \\
\mathrm{N}=102\end{array}$ & $\begin{array}{l}\text { Omnivore } \\
\mathrm{N}=100\end{array}$ & $\mathrm{p}$ value \\
\hline Body mass index $\left(\mathrm{kg} / \mathrm{m}^{2}\right)$ & $23.2(4.1)$ & $24.4(3.9)$ & $26.4(4.7)^{b, c}$ & $<0.001$ \\
\hline Waist circumference $(\mathrm{cm})$ & $79.7(11.0)$ & $81.7(10.7)$ & $86.5(12.9)^{b, c}$ & 0.001 \\
\hline Fat mass (\%) & $27.1(9.2)$ & $30.2(8.2)$ & $32.6(9.1)^{\mathrm{b}}$ & 0.001 \\
\hline Systolic BP (mmHg) & $115(14)$ & $118(16)$ & $119(14)$ & 0.328 \\
\hline Diastolic BP (mmHg) & $72(9)$ & $73(10)$ & $74(10)$ & 0.501 \\
\hline Plasma glucose (mg/dL) & $91.8(7.9)$ & $92.3(7.4)$ & $94.6(10.1)$ & 0.076 \\
\hline Fasting insulin ${ }^{\mathrm{a}}(\mu \mathrm{UI} / \mathrm{mL})$ & $6.5(1.8)$ & $7.4(1.7)$ & $9.2(1.7)^{b, c}$ & $<0.001$ \\
\hline HOMA-IR & $1.5(1.8)$ & $1.7(1.7)$ & $2.1(1.8)^{\mathrm{b}, \mathrm{c}}$ & $<0.001$ \\
\hline Total cholesterol (mg/dL) & $173.9(36.9)$ & $173.0(35.6)$ & $185.3(33.6)^{c}$ & 0.028 \\
\hline LDL-cholesterol (mg/dL) & $99.3(31.0)$ & $110.7(27.2)$ & $113.6(29.8)^{b, c}$ & 0.005 \\
\hline HDL-cholesterol ${ }^{\mathrm{a}}$ (mg/dL) & $51.7(1.3)$ & $49.7(1.3)$ & $50.4(1.3)$ & 0.614 \\
\hline Triglycerides $^{\mathrm{a}}(\mathrm{mg} / \mathrm{dL})$ & $96.5(1.55)$ & $92.2(1.58)$ & $93.4(1.68)$ & 0.831 \\
\hline C-reactive protein (mg/L) & $0.5(0.4-1.3)$ & $0.8(0.4-1.7)$ & $1.1(0.6-2.2)$ & 0.007 \\
\hline Lipopolysaccharides (ng/mL) & $31.8(24.8-45.9)$ & $33.5(16.2-51.2)$ & $39.5(23.5-54.7)$ & 0.008 \\
\hline Interleukin-10 (pg/mL) & $0.3(0.2-0.5)$ & $0.3(0.05-0.5)$ & $0.3(0.05-0.5)$ & 0.402 \\
\hline TNF-a (pg/mL) & $2.7(1.7-3.6)$ & $2.9(1.5-5.0)$ & $2.9(1.9-4.5)$ & 0.423 \\
\hline TNF-a/IL-10 & $7.3(4.5-13.1)$ & $10.5(5.6-18.4)$ & $11.7(6.5-27.2)$ & 0.015 \\
\hline E-selectin $(\mathrm{pg} / \mathrm{mL})$ & $28.5(17.5-53.5)$ & $34.1(21.0-48.4)$ & $39.4(23.5-54.7)$ & 0.110 \\
\hline
\end{tabular}

BP blood pressure, HOMA-IR insulin resistance index, TNF- $a$ tumor necrosis factor-alpha, IL-10 interleukin-10

a Log-transformed for analysis; values were back-transformed to return to the natural scale. ANOVA followed by Bonferroni post hoc test or Kruskal-Wallis test

b Versus strict vegetarian

c Versus lacto-ovo-vegetarian

while among the Bacteroidetes an expected higher proportion of Prevotella. These findings suggest that exposure to animal foods could favor a pro-inflammatory intestinal environment, favoring endotoxemia, systemic inflammation and insulin resistance that are involved in the deterioration of the cardiometabolic risk profile. 
In agreement with other studies [1-4, 30], the Brazilian strict vegetarian Adventists exhibited a low-risk cardiometabolic profile, particularly when compared to the omnivores. Lower frequencies of obesity, hypertension, and pre-diabetes were observed in the strict vegetarians, similar to the results of the adventist health study- $2[3$, 4] and also coherent with reports of fewer cardiovascular events in strict vegetarians $[30,39,40]$. Their food preferences-vegetables, fruits, and whole grains-are rich in fibers and micronutrients, which contribute to reduce oxidative stress, an underlying mechanism of these diseases [41]. Fiber-derived short-chain fatty acids (SCFA), mainly butyrate, acetate, and propionate, are facilitated by the presence of certain commensal bacteria that belong to phylum Firmicutes [42-44].

Our findings of lower proportion of Firmicutes and higher proportion of Bacteroidetes in strict vegetarians compared to omnivores may not be attributed to the differences in body adiposity of the participants. In fact, a meta-analysis did not confirm previous assumptions, based on from animal and human studies, that obesity was associated with an increased F/B ratio [19, 45].

Analyzing particularly the Firmicutes subpopulations, we found that strict vegetarians had an increased abundance of the two most recognized butyrate-producing bacteria-Roseburia and Faecalibacterium [43, 46] in comparison with omnivores. Butyrate is a major energy source for colonocytes and promotes the expression of tight junction proteins, enhancing the intestinal barrier function and consequently, it protects against the LPS translocation [43, 47]. Our finding of lower LPS concentration in the strict vegetarian group is coherent with these effects. Also, the anti-inflammatory action of butyrate due to the nuclear factor $\mathrm{\kappa B}$ inhibition in colonic cells $[43,46]$ is supported by our results since lower values of inflammatory markers, CRP and TNF- $\alpha / \mathrm{IL}-10$ ratio, were detected in the same group. However, our study design precluded establishing cause-effect relationship. Therefore, despite belonging to the Firmicutes phylum, Roseburia and Faecalibacterium genera were shown to be associated with a beneficial metabolic profile in our vegetarians, characterized by lower body adiposity and better lipid profile and insulin resistance index, which are along the same line of previous reports $[16,48]$.

Bacteroidetes were relatively more frequent in the microbiota of strict vegetarians than in omnivores, and their subpopulations were mainly composed of the genera Prevotella and Bacteroides. A higher Prevotella/Bacteroides ratio was seen in the strict vegetarians. These findings are consistent with others that reported higher Prevotella abundance in individuals with a plant-based diet and predominance of Bacteroides in non-vegetarians $[15,16,49,50]$. Also, investigations on the interaction between long-term dietary patterns and microbiota using genera clusters found an association of Prevotella enterotype with fiber-enriched diets, as well as Bacteroides enterotype with protein and animal fat [15]. One study is in disagreement, since no significant difference between the gut microbiota composition of vegetarians and omnivores was observed [47].

Our observations of higher LPS, CRP, TNF- $\alpha / \mathrm{IL}-10$ ratio, and HOMA-IR values in the omnivorous group reinforce previous hypothesis that a saturated fatenriched diet could induce inflammation and insulin resistance $[7,26,28]$. We speculate that exposure to animal foods could have contributed to alter the gut microbiota composition favoring an increase in LPS and generating endotoxemia. LPS are present in outer membrane of gram-negative bacteria and its ability to reduce the expression of tight junction proteins and increase intestinal permeability were demonstrated [29]. Also, there is evidence that microbiota-derived LPS in circulation, by binding to TLR4, trigger inflammation, deteriorate insulin signaling, and cause metabolic disturbances [7, 51, 52]. TLR4-deficient mice were recognized as resistant to the inflammatory activation induced by obesity or free fatty acids and protected from insulin resistance [52]. We suppose that, in the omnivorous participants, animal food consumption could have favored an enrichment of gram-negative bacteria, increased gut permeability and activation of immune response. Their higher levels of some inflammatory markers corroborate for this pathophysiological mechanism, anticipating the alterations in traditional cardiovascular risk factors. Actually, we detected an overrepresentation in some OTUs (increased Succinivibrio and Halomonas abundances) from the Proteobacteria phylum, which is known as a major group of gram-negative bacteria that prefer proteins as main energy source [49]. A similar result has already been reported in another study in which Proteobacteria were more abundant in European children fed with western diet compared to Africans fed predominantly with vegetarian diet [16].

Also, overrepresentation of class Bacilli, belonged to Firmicutes phylum, was verified among omnivores, which could be somehow unexpected since these bacteria are not gram-negative. However, our result is concordant with a report of an association of high abundance of Bacilli with the western diet [53] and diabetes [54], both conditions associated with low-grade inflammation and insulin resistance. Inflammatory markers and HOMA-IR were, in fact, higher in our omnivorous participants compared to vegetarians.

In lacto-ovo-vegetarians, we observed a higher proportion of Firmicutes and among these, of the Faecalibacterium genus. This finding was previously reported 
[50] and it was suggested that dairy products and eggs might be substrates for these bacteria [55]. Since these participants were exposed to this kind of animal foods and an intermediate cardiometabolic risk level, between the strict vegetarians and omnivores, was expected. Despite clinical parameters within the normal ranges, they already exhibited signs of a pro-inflammatory and reduced insulin sensitivity condition.

The main limitation is related to the cross-sectional design impeding the establishment of temporal or causal relationships. Also, the lack of detailed nutrient information inquiry made our study even more speculative. On the other hand, our study has the strength of reporting data on the gut microbiota at a deep level in a considerable number of individuals consuming with distinct dietary patterns. As far as we know, this approach is unique in South America countries. These data were associated with traditional risk factors and emergent cardiometabolic markers, suggesting possible mechanisms by which diet-mediated bacteria could participate in the genesis of prevalent diseases linked by the insulin resistance.

We called attention to the importance of gut microbiota assessment for understanding how diet participates in the pathogenesis of cardiometabolic diseases. Whether deeper taxonomic classification could provide clues in the investigation of the pathophysiological mechanisms of these complex diseases requires further investigation.

In conclusion, our data support that there are differences in gut microbiota composition of individuals consuming distinct types of diet, who differ according to their inflammatory and metabolic profiles. Based on the findings relative to bacteria abundances and on their recognized actions in the metabolism, we suggest that exposure to animal foods may favor an intestinal environment which could trigger systemic inflammation and insulin resistance-dependent metabolic disorders.

\section{Authors' contributions}

Conceived and designed the study: SRGF, ACP. Performed the data collection: ACFM, EPG. Analyzed the data: ACFM, GRF, BAP, SRGF. Contributed to the writing of the manuscript: ACFM, GRF, BAP, SRGF, EPG, ACP. All authors read and approved the final manuscript.

\section{Author details}

${ }^{1}$ Department of Epidemiology, School of Public Health, University of Sao Paulo, Av. Dr. Arnaldo, 715, Sao Paulo, SP Zip code 01246-904, Brazil. ${ }^{2}$ Department of Preventive Medicine, Federal University of Sao Paulo, Rua Botucatu, 720, Sao Paulo, SP Zip code 04023-900, Brazil. ${ }^{3}$ Oswaldo Cruz Foundation, René Rachou Research Center, Av. Augusto de Lima, 1715, Belo Horizonte, MG Zip code 30190-002, Brazil. ${ }^{4}$ Laboratory of Genetics and Molecular Cardiology, Heart Institute, Medical School, University of Sao Paulo, Av. Dr. Eneas de Carvalho Aguiar, 44, $10^{\circ}$. andar, Sao Paulo, SP Zip code 05403-000, Brazil.

\section{Acknowledgements}

Authors thank the Fapesp, Advento Study Group and participants. Members of the Advento Study Group: IJM Bensenor, PA Lotufo, KRM Gomes, LCB Soares, V Kunz, NV Silva, LA Portes, DT Kanno, LF Sella, R França,
MC Teixeira, S Gasparini, EOL Ferreira, B Bonifácio, TC Souza, FM Diaz, SCC Dammann, IR Pinheiro, WFS Costa, DMS Larchert, DF Nunes, JS Amorim, EM Reis, IP Manfrim, NV Ferreira, JLV Passos, E Barreto.

\section{Competing interests}

The authors declare that they have no competing interests.

\section{Availability of data and materials}

The $16 \mathrm{~S}$ ribosomal DNA sequences are available under study Accession PRJEB19103.

\section{Consent for publication \\ Not applicable.}

\section{Ethics approval and consent to participate}

The Institutional Ethical Committee of University of São Paulo—School of Public Health (CEP/FSP/154.416) and University Hospital (CEP/HU/1226.12)— approved the protocol and participants signed informed consent form.

\section{Funding}

The present study was supported by FAPESP (2012/12626-9 and 2012/03880-9).

\section{Publisher's Note}

Springer Nature remains neutral with regard to jurisdictional claims in published maps and institutional affiliations.

Received: 23 May 2017 Accepted: 5 August 2017

Published online: 15 August 2017

\section{References}

1. Sabaté J, Wien M. A perspective on vegetarian dietary patterns and risk of metabolic syndrome. Br J Nutr. 2015;113(S2):S136-43.

2. Tonstad S, Butler T, Yan R, Fraser GE. Type of vegetarian diet, body weight, and prevalence of type 2 diabetes. Diabetes Care. 2009;32(5):791-6.

3. Pettersen BJ, Anousheh R, Fan J, Jaceldo-Siegl K, Fraser GE. Vegetarian diets and blood pressure among white subjects: results from the adventist health study-2 (AHS-2). Public Health Nutr. 2012;15(10):1909-16.

4. Orlich MJ, Singh PN, Sabaté J, et al. Vegetarian dietary patterns and mortality in adventist health study 2. JAMA Intern Med. 2013;173(13):1230-8.

5. Calder PC, Ahluwalia N, Albers R, et al. A consideration of biomarkers to be used for evaluation of inflammation in human nutritional studies. $\mathrm{Br} J$ Nutr. 2013;109(S1):S1-34.

6. Jung UJ, Choi MS. Obesity and its metabolic complications: the role of adipokines and the relationship between obesity, inflammation, insulin resistance, dyslipidemia and nonalcoholic fatty liver disease. Int J Mol Sci. 2014;15(4):6184-223.

7. Cani PD, Amar J, Iglesias MA, et al. Metabolic endotoxemia initiates obesity and insulin resistance. Diabetes. 2007:56:1761-72.

8. Zupancic ML, Cantarel BL, Liu Z, et al. Analysis of the gut microbiota in the old order Amish and its relation to the metabolic syndrome. PLoS ONE. 2012;7(8):e43052. doi:10.1371/journal.pone.0043052.

9. Koeth RA, Wang Z, Levison BS, et al. Intestinal microbiota metabolism of I-carnitine, a nutrient in red meat, promotes atherosclerosis. Nat Med. 2013;19(5):576-85.

10. Bäckhed F, Ding H, Wang T, et al. The gut microbiota as an environmental factor that regulates fat storage. Proc Natl Acad Sci USA. 2004;101(44):15718-23.

11. Ley RE, Backed F, Turnbaugh P, Lozupone CA, Knight RD, Gordon Jl. Obesity alters gut microbial ecology. PNAS. 2005;102(31):11070-5.

12. Kim KA, Gu W, Lee IA, Joh EH, Kim DH. High fat diet-induced gut microbiota exacerbates inflammation and obesity in mice via the TLR4 signaling pathway. PLOS ONE. 2012;7(10):e47713. doi:10.1371/journal. pone.0047713.

13. Greer RL, Dong X, Moraes ACF, Zielke RA, Fernandes GR, Peremyslova $E$, Vasquez-Perez $S$, et al. Akkermansia muciniphila mediates negative 
effects of IFNy on glucose metabolism. Nat Commun. 2016;7:13329 doi:10.1038/ncomms13329.

14. Le Huërou-Luron I, Blat S, Boudry G. Breast-v. formula-feeding: impacts on the digestive tract and immediate and long-term health effects. Nutr Res Rev. 2010;23(1):23-36.

15. Wu GD, Chen J, Hoffmann C, et al. Linking long-term dietary patterns with gut microbial enterotypes. Science. 2011;334(6052):105-8.

16. de Filippo C, Cavalieri D, Di Paola M, et al. Impact of diet in shaping gut microbiota revealed by a comparative study in children from Europe and rural Africa. Proc Natl Acad Sci USA. 2010;107(33):14691-6.

17. Sonnenburg JL, Bäckhed F. Diet-microbiota interactions as moderators of human metabolism. Nature. 2016;535:56-64.

18. Raymond $F$, Ouameur AA, Déraspe $M$, et al. The initial state of the human gut microbiome determines its reshaping by antibiotics. ISME J. 2015. doi:10.1038/ismej.2015.148.

19. Finucane MM, Sharpton TJ, Laurent TJ, Pollard KS. A taxonomic signature of obesity in the microbiome? Getting to the guts of the matter. PLoS ONE. 2014;9:e84689. doi:10.1371/journal.pone.0084689.

20. Arumugam M, Raes J, Pelletier E, et al. Enterotypes of the human gut microbiome. Nature. 2011:473(7346):174-80.

21. Moraes ACF, Fernandes GR, Silva IT, Almeida-Pititto B, Gomes EP, Pereira AC, Ferreira SRG. Enterotype may drive the dietary-associated cardiometabolic risk factors. Front Cell Infect Microbiol. 2017;7:47. doi:10.3389/ fcimb.2017.00047.

22. Luthold RV, Fernandes GR, Moraes ACF, Folchetti LGD, Ferreira SRG. Gut microbiota interactions with the immunomodulatory role of vitamin $D$ in normal individuals. Metabolism. 2017:69:76-86.

23. Kim MS, Hwang SS, Park EJ, Bae JW. Strict vegetarian diet improves the risk factors associated with metabolic diseases by modulating gut microbiota and reducing intestinal inflammation. Environ Microbiol Rep. 2013;5(5):765-75. doi:10.1111/1758-2229.12079.

24. Kong LC, Holmes B, Cotillard A, et al. Dietary patterns differently associate with inflammation and gut microbiota in overweight and obese subjects. PLoS ONE. 2014;9(10):e109434. doi:10.1371/journal.pone.0109434.

25. Conlon MA, Bird AR. The impact of diet and lifestyle on gut microbiota and human health nutrients. 2015;7(1):17-44. doi:10.3390/nu7010017.

26. Lee CY. The effect of high-fat diet-induced pathophysiological changes in the gut on obesity: what should be the ideal treatment? Clin Transl Gastroenterol. 2013;4(7):e39. doi:10.1038/ctg.2013.11.

27. Moraes ACF, Silva IT, Almeida-Pititto B, Ferreira SRG. Microbiota intestinal e risco cardiometabólico: mecanismos e modulação dietética. Arq $\mathrm{Br}$ Endocrinol Metab. 2014;58(4):317-27.

28. Guo X, Li J, Tang R, Zhang G, Zeng H, Wood RJ, Liu Z. High fat diet alters gut microbiota and the expression of paneth cell-antimicrobial peptides preceding changes of circulating inflammatory cytokines. Mediators Inflamm. 2017:2017:9474896. doi:10.1155/2017/9474896.

29. Boulangé CL, Neves AN, Chilloux J, Nicholson JK, Dumas M-E. Impact of the gut microbiota on inflammation, obesity, and metabolic disease. Genome Med. 2016:8:42. doi:10.1186/s13073-016-0303-2.

30. Tonstad S, Stewart K, Oda K, Batech M, Herring RP, Fraser GE. Vegetarian diets and incidence of diabetes in the adventist health study-2. Nutr Metab Cardiovasc Dis. 2013;23(4):292-9.

31. Orlich MJ, Fraser GE. Vegetarian diets in the adventist health study 2: a review of initial published findings. Am J Clin Nutr. 2014;100(1):353S-8S. doi:10.3945/ajcn.113.071233.

32. American Diabetes Association. Classification and diagnosis of diabetes Diabetes Care. 2015:38(S1):S8-16. doi:10.2337/dc15-S005.

33. Matthews DR, Hosker JP, Rudenski AS, Naylor BA, Treacher DF, Turner RC. Homeostasis model assessment: insulin resistance and beta-cell function from fasting plasma glucose and insulin concentrations in man. Diabetologia. 1985;28:412-9.

34. Hanson RL, Pratley RE, Bogardus C, et al. Evaluation of simple indices of insulin sensitivity and insulin secretion for use in epidemiologic studies. Am J Epidemiol. 2000;151:190-8.

35. Bonora E, Targher G, Alberiche M, et al. Homeostasis model assessment closely mirrors the glucose clamp technique in the assessment of insulin sensitivity. Diabetes Care. 2000;23:57-63.
36. Goswami B, Rajappa M, Mallika V, Deepak KS, Kumar S. TNF-a/LL-10 ratio and $\mathrm{C}$-reactive protein as markers of the inflammatory response in CAD-prone North Indian patients with acute myocardial infarction. Clin Chimica Acta. 2009;408:14-8.

37. Caporaso JG, Lauber CL, Walters W, et al. Ultra-high-throughput microbial community analysis on the Illumina HiSeq and MiSeq platforms. ISME J. 2012;6(8):1621-4.

38. Love Ml, Anders S, Huber W. Moderated estimation of fold change and dispersion for RNA-seq data with DESeq2. Genome Biol. 2014;15:550.

39. Glick-Bauer M, Yeh M-C. The health advantage of a vegan diet: exploring the gut microbiota connection. Nutrients. 2014:6:4822-38.

40. Yokoyama Y, Barnard ND, Levin SM, Watanabe M. Vegetarian diets and glycemic control in diabetes: a systematic review and metaanalysis. Cardiovasc Diagn Ther. 2014;4(5):373-82. doi:10.3978/j. issn.2223-3652.2014.10.04.

41. Savini I, Catani M, Evangelista D, Gasperi V, Avigliano L. Obesity-associated oxidative stress: strategies finalized to improve redox state. Int J Mol Sci. 2013;14:10497-538.

42. Kabeerdoss J, Shobana DR, Regina MR, Ramakrishna BS. Faecal microbiota composition in vegetarians: comparison with omnivores in a cohort of young women in southern India. Br J Nutr. 2012;108(6):953-7.

43. Canani RB, Costanzo MD, Leone L, Pedata M, Meli R, Calignano A. Potential beneficial effects of butyrate in intestinal and extraintestinal diseases. World J Gastroenterol. 2011;17(12):1519-28.

44. Hartstra AV, Bouter KEC, Backhed F, Nieuwdorp M. Insights into the role of the microbiome in obesity and type 2 diabetes. Diabetes Care. 2015;38:159-65.

45. Angelakis E, Armougom F, Million M, Raoult D. The relationship between gut microbiota and weight gain in humans. Future Microbiol. 2012;7(1):91-109.

46. Miquel S, Martín R, Rossi O, et al. Faecalibacterium prausnitzii and human intestinal health. Curr Opin Microbiol. 2013;16(3):255-61.

47. Liszt K, Zwielehner J, Handschur M, Hippe B, Thaler R, Haslberger AG. Characterization of bacteria, clostridia and Bacteroides in faeces of vegetarians using QPCR and PCR-DGGE fingerprinting. Ann Nutr Metab. 2009;54(4):253-7.

48. Martínez I, Lattimer JM, Hubach $\mathrm{KL}$, et al. Gut microbiome composition is linked to whole grain-induced immunological improvements. ISME J. 2013;7(2):269-80

49. Ruengsomwong S, Korenori Y, Sakamoto N, Wannissorn B, Nakayama J, Nitisinprasert S. Senior thai fecal microbiota comparison between vegetarians and non-vegetarians using PCR-DGGE and real-time PCR. J Microbiol Biotechnol. 2014;24(8):1026-33.

50. Ferrocino I, Di Cagno R, De Angelis M, et al. Fecal microbiota in healthy subjects following omnivore, vegetarian and vegan diets: culturable populations and rRNA DGGE profiling. PLoS ONE. 2015;10(6):e0128669. doi:10.1371/journal.pone.0128669.

51. Liang H, Hussey SE, Sanchez-Avila A, Tantiwong P, Musi N. Effect of lipopolysaccharide on inflammation and insulin action in human muscle. PLoS ONE. 2013;8(5):e63983. doi:10.1371/journal.pone.0063983.

52. Caricilli AM, Saad MJ. The role of gut microbiota on insulin resistance. Nutrients. 2013;5:829-51.

53. Turnbaugh PJ, Ridaura VK, Faith JJ, Rey FE, Knight R, Gordon JI. The effect of diet on the human gut microbiome: a metagenomic analysis in humanized gnotobiotic mice. Sci Transl Med. 2009;1(6):6ra14. doi:10.1126/scitransImed.3000322.

54. Larsen N, Vogensen FK, van den Berg FW, Nielsen DS, Andreasen AS, Pedersen BK, Al-Soud WA, et al. Gut microbiota in human adults with type 2 diabetes differs from non-diabetic adults. PLoS ONE. 2010;5(2):e9085. doi:10.1371/journal.pone.0009085.

55. Khan MT, Duncan SH, Stams AJM, van Dijl JM, Flint HJ, Harmsen HJM. The gut anaerobe Faecalibacterium prausnitzii uses an extracellular electron shuttle to grow at oxic-anoxic interphases. ISME J. 2012;6(8):1578-85. 